SUPPORTING INFORMATION

\title{
Effective Biological DeNOx of Industrial Flue Gas by the Mixotrophic Cultivation of an Oil-Producing Green Alga
} Chlorella sp. C2

Weixian Chen ${ }^{1,3 \#}$, Shanshan Zhang ${ }^{1 \#}$, Junfeng Rong ${ }^{2}$, Xiang $\mathrm{Li}^{4}$, Hui Chen ${ }^{1}$, Chenliu $\mathrm{He}^{1}$, Qiang Wang ${ }^{1, *}$

${ }^{1}$ Key Laboratory of Algal Biology, Institute of Hydrobiology, Chinese Academy of Sciences, Wuhan 430072, Hubei, China

${ }^{2}$ SINOPEC Research Institute of Petroleum Processing, Beijing, China

${ }^{3}$ University of Chinese Academy of Sciences, Beijing 100094, China

${ }^{4}$ College of Life Sciences, Northeast Forestry University, Harbin 150040, Heilongjiang, China

* Correspondence: Wang, Qiang, Key Laboratory of Algal Biology, Institute of Hydrobiology, Chinese Academy of Sciences, 7 South Donghu Rd., Wuhan, Hubei Province, 430072 China, e-mail: qwang@ihb.ac.cn.

\# These authors contributed equally to this work

The number of pages is 2 , and the number of figures is 1 . 


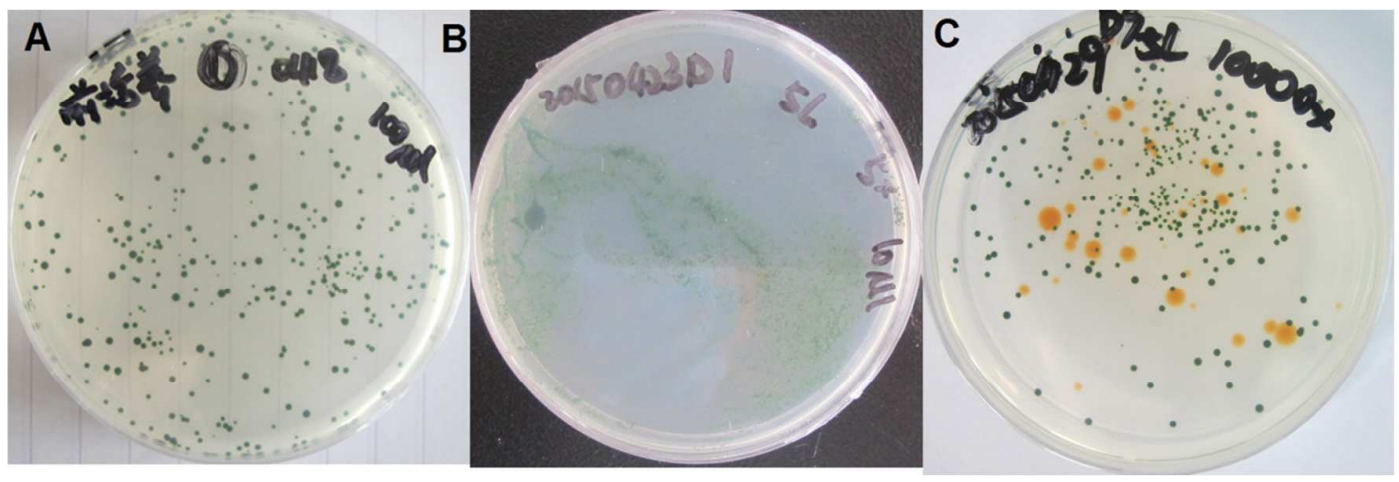

Figure S1. The detection of microbial contamination in the $5 \mathrm{~L}$ culture systems with very light contamination by the end of cultivation.

The Chlorella sp. C2 cultures were randomly sampled and spread on regular BG11 solid plate to identify the possible microbial contaminations after more than 10 days of incubation. (A), Pre-culture sample for inoculation (April 18, 2015); (B), sample at the first day of cultivation (April 23, 2015); (C), sample with very light contamination by the end of cultivation (April $29,2015)$. 